\title{
Robust and Effective Banknote Recognition Model for Aiding Visual Impaired People
}

\author{
Asfaw Alene Shefraw \\ Ethiopian Institute of Textile and Fashion technology, Bahir Dar Universiity, Bahir Dar, Ethiopia
}

\begin{tabular}{l} 
Article Info \\
Article history: \\
Received Dec 27, 2020 \\
Revised Aug 9, 2021 \\
Accepted Sep 17, 2021 \\
\hline Keywords: \\
Banknote recognition system \\
Visual desiable person \\
Automated Ethiopian birr-note \\
Currency recognition system \\
Denomination specific ROI
\end{tabular}

\begin{abstract}
Visual disabled Ethiopians find great difficulty in recognizing banknotes. Each Ethiopian banknote has an identical feel, with no Braille markings, irregular edges, or other tangible features that make it easily recognizable by blind persons. In Ethiopia, there's only one device available that will assist blind people to acknowledge their notes. Internationally, there are devices available; however, they're expensive, complex, and haven't been developed to cater to Ethiopian currency. Because of these facts, visually impaired people may suffer from recognizing each folding money. This fact necessitates a higher authentication and verification system that will help visually disabled people to simply identify and recognize the banknotes. This paper presents a denomination-specific component-based framework for a banknote recognition system. Within the study, the dominant color of the banknotes was first identified and so the exclusive feature for every denomination-specific ROI was calculated. Finally, the Colour-Momentum, dominant color, and GLCM features were calculated from each denomination-specific ROI. Designing the recognition system by thereby considering the denominationspecific ROI is simpler as compared to considering the entire note in collecting more class-specific information and robust in copying with partial occlusion and viewpoint changes. The performance of the proposed model was verified by using a larger dataset of which containing banknotes in several conditions including occlusion, cluttered background, rotation, and changes of illumination, scaling, and viewpoints. The proposed algorithm achieves a 98\% recognition rate on our challenging datasets.
\end{abstract}

Copyright (C) 2021 Institute of Advanced Engineering and Science. All rights reserved.

\section{Corresponding Author:}

Asfaw Alene Shefraw,

Ethiopian institute of textile and Fashion technology,

Ethiopian Institute of Textile and Fashion Technology, Bahir Dar University

Amhara, Bahir Dar, Ethiopia

Email: asa4ever2002@gmail.com

\section{INTRODUCTION}

According to World Blind Union (WBU), there were 61 million visually impaired people around the world in 2002, which is about $2.6 \%$ of the total population. Among these people, 124 million were registered to have low vision and 37 million were blind $[30,16]$. As a matter of fact, visually impaired people face a number of challenges when interacting with the environment. Due to this, such information is visually encoded in their daily life. One specific difficulty that a visually disabled person will face is the task of identifying the value of the notes he or she is holding [20]. Currently, printed denominations of Ethiopian currencies are 1Birr, 5-Birr, 10-Birr, 50-Birr, and 100-Birr. With a few recent exceptions, all of the banknotes are different in size, a shape that is printed through intaglio printing, and color which is inaccessible to people who are blind or significantly visually impaired.

The national bank of Ethiopia which is responsible to print hard currencies in the country uses both intrinsic as well as extrinsic features to design the banknotes. The extrinsic properties accommodate the size, 
width, color, etc. whereas the intrinsic properties include security thread, I.D. mark, number panel, etc. Extrinsic properties do not seem to be enough to acknowledge whether the note is original or fake. Also, currency may get damaged during transportation or exchange. The change within the nature of a picture may be well understood and improved with the assistance of image processing techniques. It enhances a number of the protection features embedded within the image for human interpretation.

One local study that the researcher considers of particular relevance to the current study (because of the similarity within the objectives and system setup) was published by Jegnaw and Yaregal [15]. Within the study, they proposed an Ethiopian banknote recognition system using dominant color, the correlation of the hue, saturation, and intensity of the HSV color, SURF, and widen strip ROI. Within the study, a template matching method was considered to spot the patterns of every feature. In step with the experimental analysis, $90.42 \%$ recognition rates for genuine Ethiopian currency with a median time interval of 1.68 seconds per banknote were achieved. Nevertheless, the study achieves a better result, using the color feature for originality check isn't valid and robust. Moreover, the template matching classifiers aren't invariant to intensity value change [19].

Another research project which is particularly relevant to this present study was published by Hasanuzzaman et al. [12]. In the study, they proposed a method based on component-based SURF for the recognition of U.S. dollar banknotes. The study aimed to design a model to be used by visually impaired persons in uncontrolled environments. Although their proposed methods are robust to several conditions, including scale variations, rotation, occlusions, wrinkling of the banknote, etc, the proposed models are not applicable within the case of the presence of severe motion blur. Moreover, their method is much more computationally expensive than the one presented in this paper. From the experimental analysis, they achieved a real recognition result of $100 \%$ when the acquired banknote image is of sufficient quality.

The artificial intelligence improvements and development of technologies have enabled great advances in the use of artificial vision for the recognition of the value of several currencies, such as Euro banknotes [5,17,28], Ethiopian banknotes [2,25], U.S. Dollars [18, 21,27,32], Rupees [26], Mexican banknotes [10], the currency of Saudi Arabia [24], etc. In their study, they propose an artificial intelligence-based fully automatic assistive technology to recognize different objects, and auditory inputs are provided to the user in real-time, which gives a better understanding to the visually impaired person about their surroundings

The banknote recognition system invariably depends on the characteristic features that constitute the banknotes and also the method used to extract the feature associated with the feature available within the notes. Additionally, different banknotes of various countries such as India, the U.S., and China have a known registered unique serial number by which features for verification and recognition are utilized. While intensive works were done to acknowledge banknotes, there are only a few studies conducted with reference to Ethiopia banknotes.

Due to this, the class-specific features of the notes are not uniformly distributed across different banknotes. While some areas of the surface cover many obvious class features, other regions have various implicit features embedded in the banknote fields. Thus, this study aims to design an Ethiopian banknote recognition system using denomination-specific ROI. It further presents a simple and robust method for the identification of the Ethiopian banknotes.

\section{RESEARCH METHOD}

\subsection{Materials}

Ethiopian banknote: Newer Versions of Ethiopian banknote bills containing values 10, 50, 100, and 200 were used throughout the course of this study. A total of 2400 notes including clean, noisy, worn, and torn were included in the process of investigation.

\subsection{Methods}

In this paper, a novel component-based banknote recognition system that uses color moments and GLCM features to achieve high recognition accuracy and handle various challenging conditions in real-world environments is proposed. Figure 1 shows the system diagram. First, the dominant color of the notes was identified. Then, the denomination-specific ROI is extracted. Next, the monetary features of each ROI are extracted by GLCM and color moments. These features are then used for training and testing the proposed model. Figure 1 describes the block diagram of the system's functionality. 


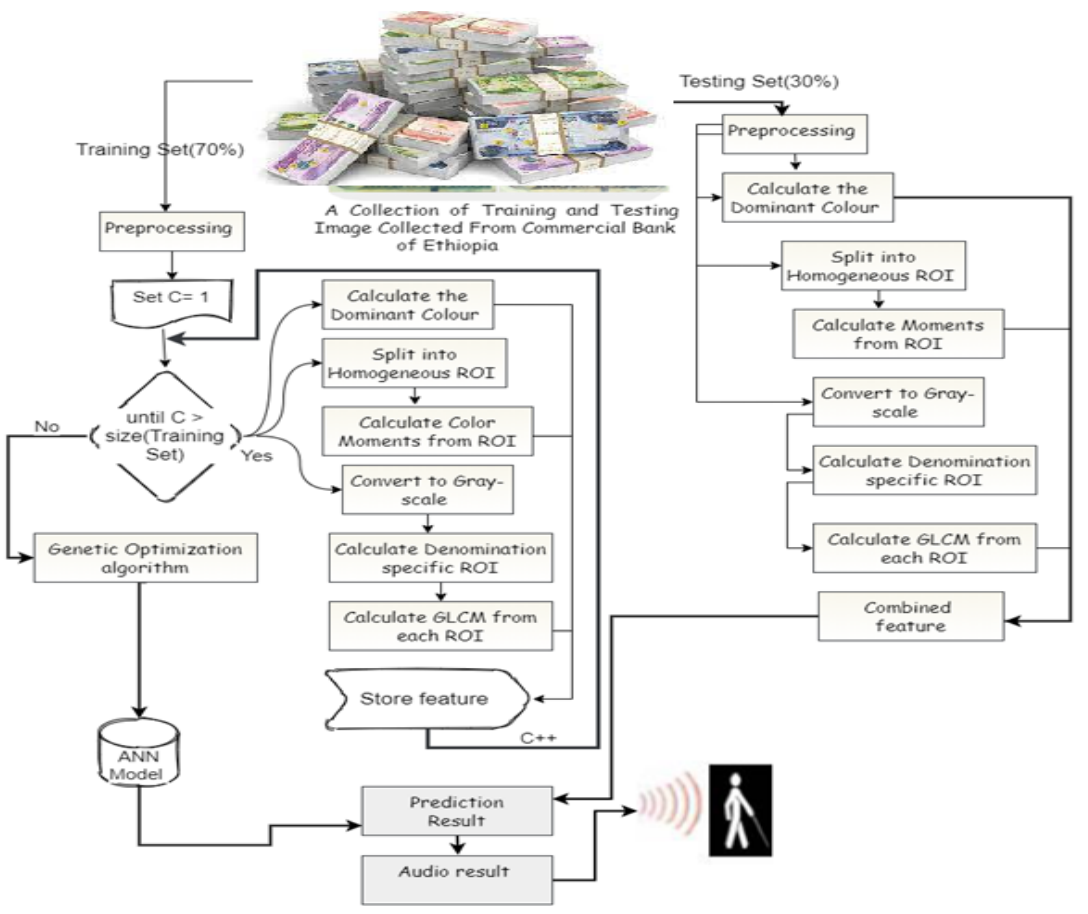

Figure 1. Block diagram of the proposed method

\subsubsection{Image Acquisition}

Image acquisition refers to the process of capturing an image of any object using a personal cell phone. In the case of this study, the image processing involved capturing the notes through a personal cell phone. The captured images were stored for further processing.

\subsubsection{Image Pre-Processing}

In these computational steps of the model, image features are preprocessed to enhance important features. In this computational phase of the model, the target image was resized to a fixed size of $224 * 315 * 3$ using neighborhood interpolation techniques to make the size of each banknote uniform [6]. To remove noise from the target banknote image and improve the quality of banknote recognition, the bilateral filter is applied because it preserves the edges and blobs as important features in banknote images. Then the contrast of the target image is improved using a Contrast Limited Adaptive Histogram Equalization (CLAHE). Finally, the background was subtracted, and the golden strip region of interest/ROI was segmented using the localized adaptive method. To speed up the input processing for each category, the proposed framework can recognize all images in a specific directory and save the results in the text file. Consequently, the target image is converted from a colored image to a grey scaled image by making use of the following equation.

Gry_img $=(0.17 * \mathrm{R}+0.62 * \mathrm{G}+0.21 * \mathrm{~B})$.

Where Gry_img is the intensity value, $R$ is the red channel value, $G$ is green channel value, and $B$ is blue channel value, and the values $0.17 \%, 0.62 \%$, and $0.21 \%$ have weight or contribution level of the red, green, and blue channels respectively which is computed through a trial-and-error method.

\subsubsection{ROI Extraction}

During this phase of the model, the image background was segmented from the foreground using threshold-based segmentation. Moreover, the denomination-specific ROI was segmented using localization techniques (see Figure 2). During this regard, the wide strip of the banknotes was cropped automatically by giving the precise locations which are in between 450 to 500 pixels. MATLAB function was employed for this operation. The pixel positions of the strip $\mathrm{x}$ and $\mathrm{y}$ as an input, are cropped images $=$ Image $(: \mathrm{x}: \mathrm{y},:)$.

\subsubsection{Feature extraction}

During these computational steps of the model, the first the dominant color of the notes was identified. Then, after the preliminary examination of notes, the ROI were identified accordingly by considering the correlation coficent of the notes with the bench mark bills of the four denominations of the notes. Lastly, the GLCM and the colour moments of each ROI were calculated. 


\section{Experimental Setup}

The dataset collected from a wide variety of environments includes notes taken under the conditions of occlusion, cluttered background, rotation, and changes of illumination, scaling, and viewpoints. Fig. 3 demonstrates four sample images from each condition. The dataset presented in our experiment is tougher as compared to any other similar study. This is because the bills contain the image which is captured under restricted or standard conditions such as occlusion, cluttered background, rotation, scaling change, and illumination change, respectively. Thus, our dataset generalizing the conditions of taking banknote images is tougher and more approximates to the real-world application environment.

\subsection{Color Features:}

In this study, the potential color space was identified through a literature review. Accordingly, both RGB and HSV color spaces were identified as a potential color spaces $[3,8,31]$. Then the performance of the candidate color space was examined. Following the banknotes, dominant color and color moments were analyzed to identify the color feature. Based on these features, the classification into their respective denominations is carried out.

Color Spaces: In this study, the performance of RGB and HSV color space was examined to design the recognition system.

\section{a) Color Moments}

To determine the color similarity between images, the color moment was measured and calculated. To calculate the moments first bill notes were divided into a number of homogeneous subblocks and then the distribution of each subblock would be calculated separately (See Figure 2). After that, the RGB channels of each subblock were converted to HSV-channel color space. Finally, the mean, standard deviation, and skewness of each color channel such as; hue, saturated, and intensity of each channel in each subblock were examined separately and concatenated as a feature. In this study, an image was characterized by a total of 54 color features obtained by taking nine features from each individual subblock. The first-order color moment called mean (, which is described as the average color in the image was calculated (See equation 2, 3, 4).

$$
\mu=\frac{1}{n} \sum_{i=1}^{n} f_{i j}
$$

Where fij is the value of the ith color component of the image pixel $\mathrm{j}$, and $\mathrm{n}$ is the number of pixels in the image.

Moment 2 - STANDARD DEVIATION: Standard deviation is derived by using Equation (3).

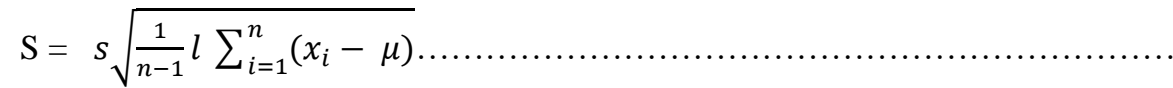

Moment 3 - SKEWNESS: Skewness is derived by using Equation (4)

$$
\mathrm{s}_{\mathrm{i}}=\sqrt[3]{\frac{1}{\mathrm{n}} \sum_{j=1}^{n}\left(\mathrm{f}_{\mathrm{ij}}-\mu\right)^{2}}
$$

\section{b) Dominating Color}

The dominant colors in Ethiopian banknotes were calculated, thereby identifying the color with the highest pixel occupancy.

\subsection{Texture Features}

The texture is a feature that represents the surface and structure of an image, or it can be defined as a regular repetition of an element or pattern on a surface $[1,2,11,14]$. The texture of an image is complex visual patterns that are composed of entities or regions with subpatterns with the characteristics of brightness, color, shape, size, etc. To extract the texture features of Ethiopian Banknotes, GLCM features were used.

\subsubsection{GLCM Feature}

A statistical method that GLCM studies is the spatial dependency between gray levels. It characterizes the texture of an image by calculating how often pairs of pixels with specific values and in a specified spatial relationship occur in an image, creating a GLCM, and then extracting statistical measures from this matrix. The extracted GLCM features including energy, homogeneity, correlation, contrast, and entropy as the texture feature to differentiate various banknotes. To extract the GLCM, six different denominations specific ROIS were segmented and labeled as blocks 1-6, and each block was quantized by an optimal gray level. After identifying the gray levels, the optimal neighboring orientation and distance were examined until better recognition performance was achieved. Then the energy, homogeneity, correlation, contrast, and entropy of each block were calculated as a feature. 


\subsubsection{Component-based Framework for Banknote Recognition}

The proposed method relied on a component-based model. It has four main advantages over the global model: 1) The denomination-specific information is not evenly distributed on the banknote. Some portions cover more obvious class-specific features while other regions are relatively similar across different classes. It would be more effective to use denomination-specific components for the banknotes recognition system. 2) A denomination-specific-based model is ready to concentrate on local and stable parts, but the pattern of a whole banknote under the geometric and photometric changes would create variation. 3) Local image features that are generated from components guarantee a higher degree of recognition compared to the global detection method. This helps to speed up the recognition process and reduce memory capacity requirements. 4) A component-based model is more robust in handling partial occlusions compared to the whole portion of the note. It is empirically impossible to require an account of all conditions which cover the spectrum of possible variations which will result from occlusions [11]. Within the denomination-specific model, individual components are detected by their corresponding detectors. Partial occlusions only affect the outputs of some of the component detectors. As long as a specific number of components is detected, the entire banknote remains ready to be recognized.

\subsubsection{Component Generation}

In the first step, each denomination can be identified through its color value in addition to its size. In other words, every note has a security feature that bankers and financial institutions use to verify its originality. Therefore, designing a banknote recognition system using the denomination-specific ROI will enhance the performance of the recognizer.

In the proposed banknote recognition model, the front side of the banknote was examined. Figure 2 shows the Ethiopian banknote images of the front sides of 10-Birr, 50-Birr, 100-Birr, and 200-Birr. The marked regions in red are the components chosen as reference regions for each banknote's ROI. For example, in the 10-Birr bill, the specific distinguishing information in the front side is the Arabic number "10", and its Ethiopic

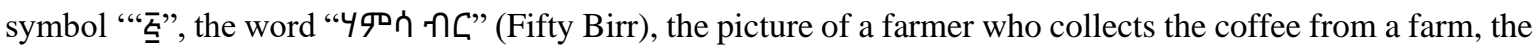
picture of a triangle which is printed using intaglio printing for aiding visually disabled people. The 10 birrs note also contains specific distinguishing information in the front side is the number "10", and its Ethiopic

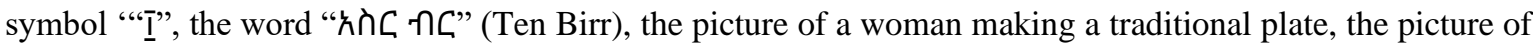
a rectangle and square is printed using intaglio printing for aiding visually disabled people. Moreover, the 10birr note contains the Golden strip ROI for a wide and narrow security thread that contains visible and invisible security features. The same principal features are seen in the other notes on both the front and back sides. Figure 2 shows the illustration of the component-based extraction technique used in this study.

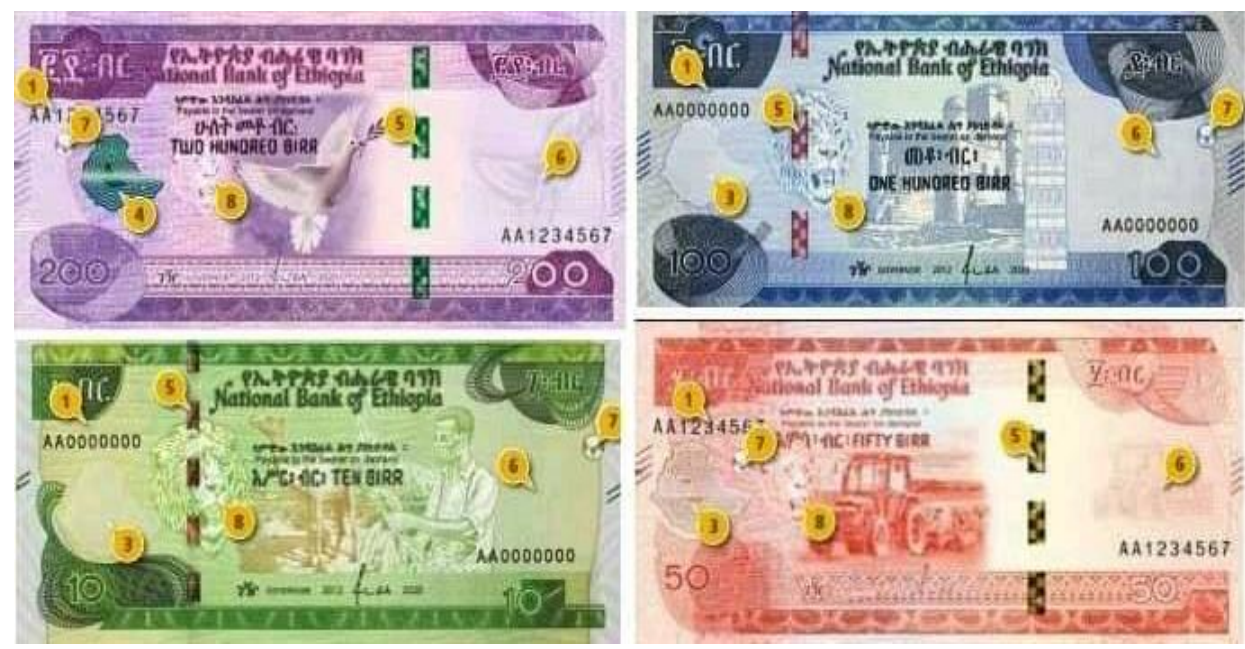

Figure 2. Reference regions with class-specific features on the bill of 100-birr notes. Each region marked in a red box is one reference region.

As shown in Figure 2, the ROI found at the middle right end side of the bill containing the shape (which is marked with red) indicates the identification mark through which the visually impaired people identify the note. The identification marks found in 10-Birr, 50-Birr, 100-Birr, and 200-Birr bills are a triangle, a rectangle, and square, one square enclosed with two rectangle shapes respectively (see figure 2).

In Ethiopian banknotes, every denomination has a unique denomination-specific identification mark on which the recognition system should rely on. In Ethiopian banknotes, the banknote serial number is found in 
twice the denomination number written through Arabic and Ethiopic numbers, the security threads are some of the security features mentioned in the study. However, also there is a security feature that is visible through Altera violet rays

\section{CLASSIFICATION}

The last layer of the proposed model is the classification layer where a machine learning approach is used. In the study, a neural network-based recognition scheme was used for currency recognition. In the study, the color moments, dominant color, and GLCM feature calculated from the bill notes were relied upon to support the classification algorithms such as ANN model Classifier (NBC) for recognizing each class of banknote.

\section{AUDIO OUTPUT GENERATION}

The output recognized text codes were recorded in the script files. Then, the study used the text-to-to-speech converter to load these files and display the audio output of text information. Visually disabled users can adjust speech rate, volume, and language according to their preferences

\section{RESULTS AND DISCUSSION}

The performance of the proposed system has been evaluated using the developed dataset of 2400 images 400-notes in each denomination. The new versions of Ethiopian banknotes were used for training and testing the proposed system. Out of the total collected dataset, $70 \%$ of the banknotes are used for training and $15 \%$ for validating and $15 \%$ for testing the proposed model. In the experiments, this research tested a database of 720 Ethiopian banknotes, which includes 6 kinds of Ethiopian banknotes (10-birr, 50-birr, and 100-birr original and fake, and 200-birr original and fake notes) (see Figure 3).

In this section, it is explained the results of the research and at the same time is given a comprehensive discussion. Results can be presented in figures, graphs, tables, and others that make the reader understand easily.

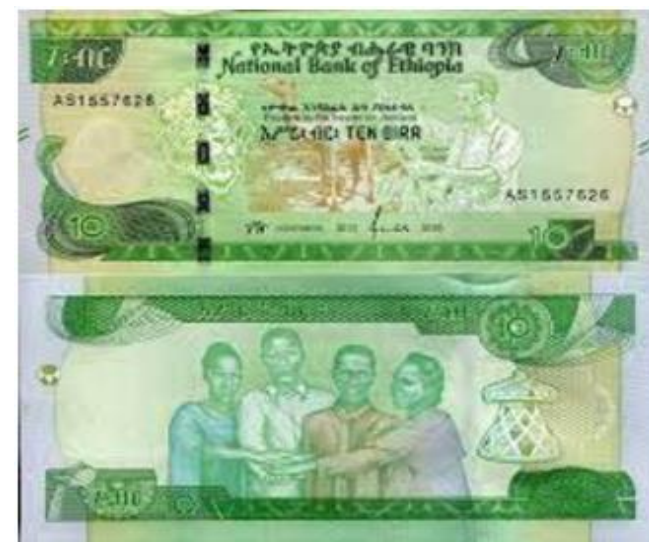

A)

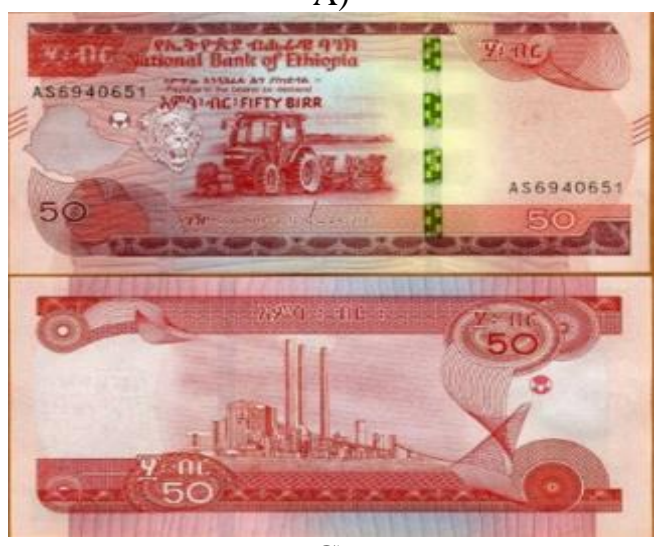

C)

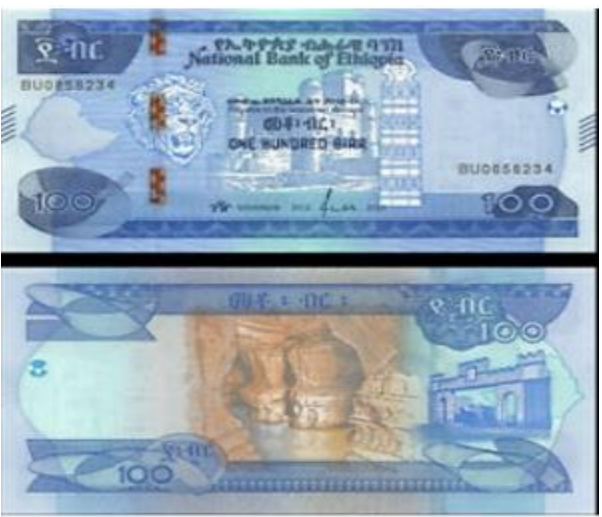

B)
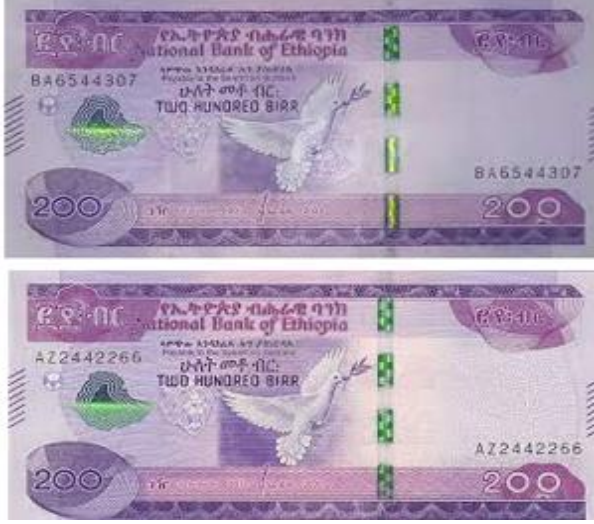

D)

Figure 3. Ethiopian banknotes A) 10-Eth Birr B) 100-Eth Birr C) Eth-Birr D) 200 scanned image 
In this study, a prototype for a banknote denomination classification system has been formulated based on the recognition of the birr notes using their intrinsic component-based color and texture features and the correlaltion coefficient between the localized widening strip ROI between the benchmark and the test image. In doing so, color moments, the dominant color, GLCM, and correlation coefficient were identified as a color features of the notes. Experiments have been conducted to assess the performance of each feature vector using an ANN classifier. The performances of each individual feature were identified separately as well as together in different arrangements.

Firstly, the brightness was adjusted by using histogram equalization techniques. Figure 4 highlights the image after applying the histogram equalization technique. Figure 4 shows an Ethiopian banknote before (Figure $4 \mathrm{a}$ ) and after (Figure $4 \mathrm{~b}$ ) contrast.

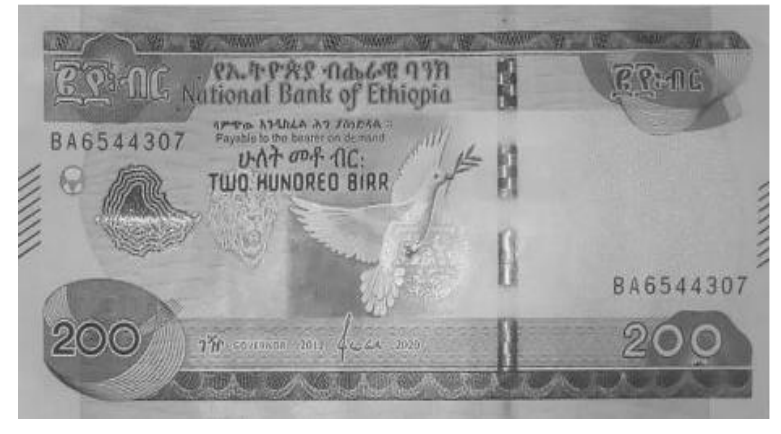

A)

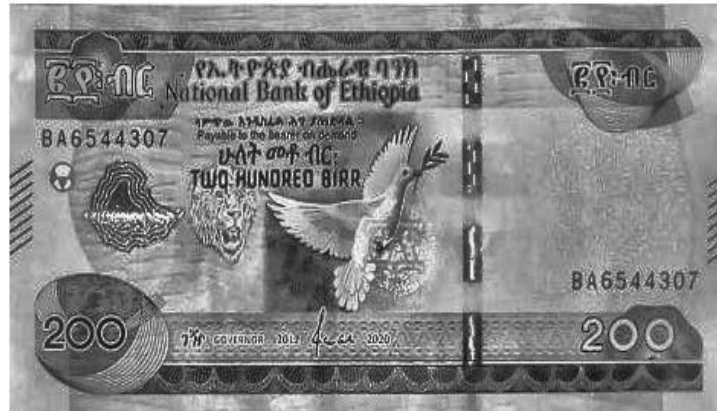

B)

Figure 4. Contrast progress states, (a)before and (b)after improvement

\subsection{The dominant color of the Ethiopian bill notes.}

After the banknote image was resized to a fixed size of $224 * 315 * 3$, the brightness and contrast were normalized, and the effects of noise were minimized using histogram equalization techniques, the study computed the dominant color feature using RGB color space. In this study, the dominant color feature was extracted from the whole banknote, and the dominant features of each note are described as shown in Table 1. As it is clearly seen in Table 1, the dominant color alone is not enough to classify different values of the banknotes. It misclassified the notes into an inaccurate class. It miss-classifies the 10-birr note wrongly to 50 and 100 to 5-notes and vice versa (Table 1 ).

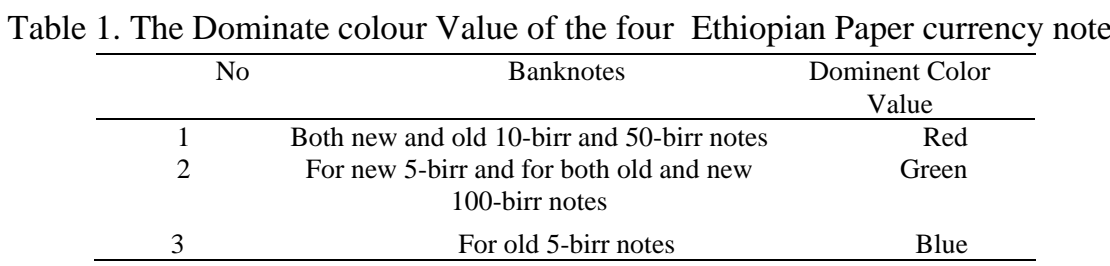

\subsection{Color space}

Images delivered by contemporary scanners or digital cameras capture the human perception of primary colors in a combination of the tri stimuli, namely, red (B), green $(G)$, and blue (B) into electrical signals. Following electronic processing of these color signals can take several different representation formats denoted as color spaces. Each color space has its own advantages and disadvantages depending on the problem being used. In the context of banknote classification, the choice of color space may have a paramount influence on the performance of procedures such as banknote feature extraction [1,2,4,31].

According to Jegnaw and Yaregal [19], the banknote denomination 5 and 100-birr notes plus 50 and 10-birr notes have the same dominant color of green and red respectively. However, as human conceptual understanding of colors, they seem totally different. These are because; the RGB color space does not correspond well to perceived differences in color. It is because there are colors that are close in RGB space, but appear very different to humans and vice versa. Furthermore, the RGB color space is sensitive to noise and cannot separate color information from luminance. On the other hand, the HSV model describes colors similarly to how the human eye tends to perceive color. It is a commonly used color space in image processing applications [2,25]. In this space, hue is used to distinguish colors, saturation is the percentage of white light added to a pure color, and value refers to the perceived light intensity. The advantage of HSV color space is that it is closer to the human conceptual understanding of colors and has the ability to separate chromatic and achromatic components. 


\subsection{Color moments}

The banknote color feature was studied using color moments, thereby using a block-based technique. In the study, the banknotes were divided into a number of blocks of the size of $112 \times 105 \times 3$. Then the performance of block-based color moments was compared with non-block-based which is by taking the entire range of the notes for calculating the color moments. The result in Table 2 shows that the recognition performances of the color moments using block-based color moments. Recognition performance of $96.1 \%$ and 98.3\% were observed while considering non-block-based (taking the entire note as a whole) and block-based for computing the coloring moments feature respectively.

Table 2. Performance of ANN using block-based color moments

\begin{tabular}{|c|c|c|c|c|c|}
\hline \multirow{6}{*}{ 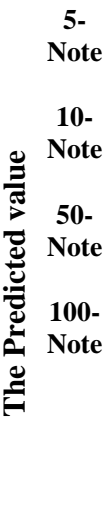 } & $\begin{array}{c}119 \\
24.8 \%\end{array}$ & $\begin{array}{c}1 \\
0.2 \%\end{array}$ & $\begin{array}{c}1 \\
0.2 \%\end{array}$ & $\begin{array}{c}0 \\
0.0 \%\end{array}$ & $\begin{array}{c}98.3 \% \\
1.7 \%\end{array}$ \\
\hline & $\begin{array}{c}0 \\
0.0 \%\end{array}$ & $\begin{array}{c}117 \\
24.4 \%\end{array}$ & $\begin{array}{c}2 \\
0.4 \%\end{array}$ & $\begin{array}{c}0 \\
0.0 \%\end{array}$ & $\begin{array}{c}98.3 \% \\
1.7 \%\end{array}$ \\
\hline & $\begin{array}{c}0 \\
0.0 \%\end{array}$ & $\begin{array}{c}2 \\
0.4 \%\end{array}$ & $\begin{array}{c}116 \\
24.2 \%\end{array}$ & $\begin{array}{c}0 \\
0.0 \%\end{array}$ & $\begin{array}{l}98.3 \% \\
1.7 \%\end{array}$ \\
\hline & $\begin{array}{c}1 \\
0.2 \%\end{array}$ & $\begin{array}{c}0 \\
0.0 \%\end{array}$ & $\begin{array}{c}1 \\
0.2 \%\end{array}$ & $\begin{array}{c}120 \\
25.0 \%\end{array}$ & $\begin{array}{c}98.4 \% \\
1.6 \%\end{array}$ \\
\hline & $\begin{array}{c}99.2 \% \\
0.8 \%\end{array}$ & $\begin{array}{c}97.5 \% \\
2.5 \%\end{array}$ & $\begin{array}{c}96.7 \% \\
3.3 \%\end{array}$ & $\begin{array}{l}100 \% \\
0.0 \%\end{array}$ & $\begin{array}{c}98.3 \% \\
1.7 \%\end{array}$ \\
\hline & $\begin{array}{c}5- \\
\text { Note }\end{array}$ & $\begin{array}{c}\text { 10- } \\
\text { Note }\end{array}$ & 50- Note & $\begin{array}{l}\text { 100- } \\
\text { Note }\end{array}$ & \\
\hline
\end{tabular}

\subsection{GLCM feature}

The texture feature of the notes was studied using a static texture feature descriptor called GLCM. To extract the component-based feature using GLCM, the RGB image is converted into a grayscale image with the contribution of each channel taken into account. Figure 5 shows the grayscale image obtained through the contribution of each channel into account. After the grayscale image is obtained, the components of the notes were segmented using the sliding window technique (see Figure 2). In this study, the GLCM feature is studied by considering a number of hypermeters such as the number of gray levels, neighboring distance, and orientation with different arrangements were studied. In doing so, a gray level of 4, 8, 12, 16, 20, 24, and 48 were used to calculate the GLCM and a better result was achieved at the gray level of 12. From the experimental studies, the study observed a performance improvement where the study used neighboring orientations of 0 , 90, and 135-degree orientations. Moreover, the performance improvements were registered while considering a long-distance neighbor as compared to the closest neighbors for studying the gray level dependency between gray levels. It is; therefore, better recognition performance was achieved while the study considered a gray level of 12, four neighboring distances with an orientation 0-degree, 90-degree, and 135-degree to study the spatial dependency between grey levels.

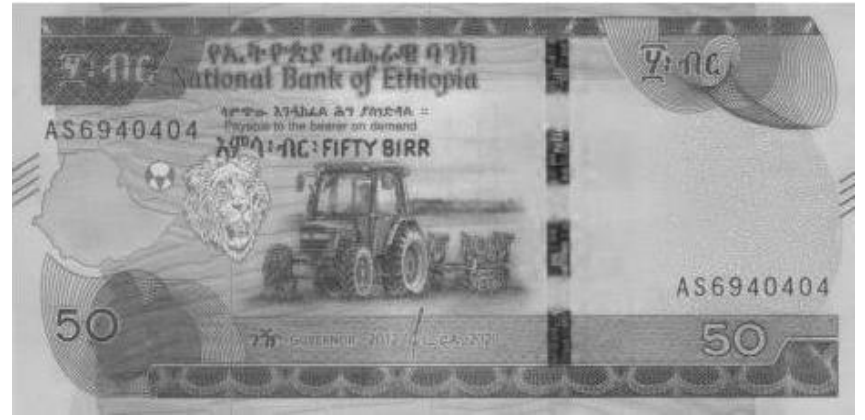

Figure 5. Ethiopia 50 birr note gray scale image

Different scholars considered different methods for designing their model for recognizing banknotes $[17,21,22,23]$. In doing so, some of them are focusing on the specific ROI, and some researchers also focusing on the entire notes to design the classification and recognition model. Calculating the GLCM feature from the entire banknote image are computationally intensive in addition to lowering the recognition performance as compared to considering the denomination-specific ROI. Therefore, the class-specific component was 
identified and extracted using the sliding window technique. In doing so, firstly, each note is subdivided into a number of class-specific six individual ROI and the GLCM of each ROI was calculated.

After calculating the GLCM matrixes by using an optimal parameter of each ROI, the contrast, correlation, energy, entropy, and homogeneity of each block were calculated separately. The performances of each block were studied separately and together in different arrangements (see Tables 3, 4).

Table 3. The performance of Component based GLCM feature

\begin{tabular}{clcc}
\hline \multirow{2}{*}{ No } & \multicolumn{1}{c}{ Feature from Calculated from } & \multicolumn{2}{c}{ Recognition performance in \% } \\
\cline { 3 - 4 } & & Testing & training \\
\hline 1 & Block 1 & 89.5 & 98 \\
2 & Block 2 & 90.4 & 98.2 \\
3 & Block 3 & 88.8 & 94.3 \\
4 & Block 4 & 90 & 95.4 \\
5 & Comined feature of Block 1,2,3,4 with & 98.8 & 100 \\
6 & an optimal arrangement & 96 & 98.8 \\
\hline
\end{tabular}

Table 4. The performance ANN using A) non-block based B) block-based GLCM feature

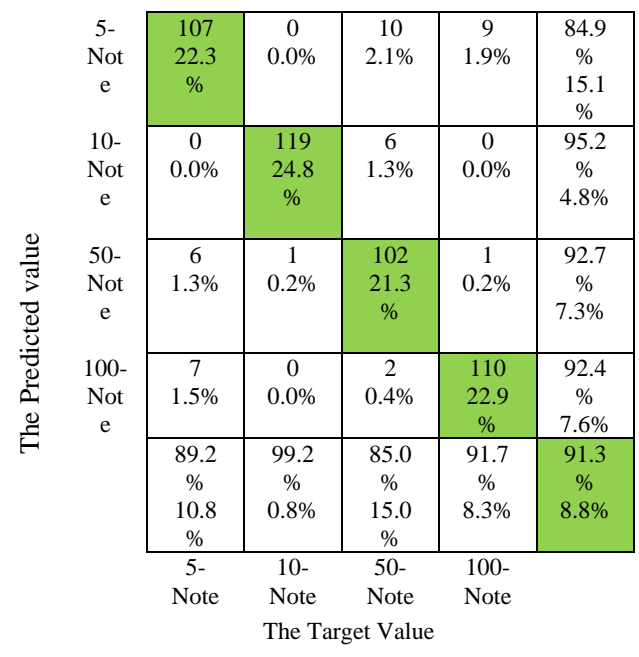

A)

\begin{tabular}{|c|c|c|c|c|c|}
\hline $\begin{array}{c}5- \\
\text { Note }\end{array}$ & $\begin{array}{c}120 \\
25.0 \%\end{array}$ & $\begin{array}{c}1 \\
0.2 \%\end{array}$ & $\begin{array}{c}0 \\
0.0 \%\end{array}$ & $\begin{array}{c}1 \\
0.2 \%\end{array}$ & $\begin{array}{c}98.4 \% \\
1.6 \%\end{array}$ \\
\hline $\begin{array}{l}10- \\
\text { Note }\end{array}$ & $\begin{array}{c}0 \\
0.0 \%\end{array}$ & $\begin{array}{c}116 \\
24.2 \%\end{array}$ & $\begin{array}{c}0 \\
0.0 \%\end{array}$ & $\begin{array}{c}0 \\
0 \%\end{array}$ & $100 \%$ \\
\hline $50-$ & 0 & 3 & 120 & 1 & $98.8 \%$ \\
\hline Note & $0.0 \%$ & $0.6 \%$ & $25.0 \%$ & $0.2 \%$ & $3.2 \%$ \\
\hline \multirow[t]{3}{*}{$\begin{array}{l}100- \\
\text { Note }\end{array}$} & $\begin{array}{c}0 \\
0.0 \%\end{array}$ & $\begin{array}{c}0 \\
0.0 \%\end{array}$ & $\begin{array}{c}0 \\
0.0 \%\end{array}$ & $\begin{array}{c}118 \\
24.6 \%\end{array}$ & $\begin{array}{l}100 \% \\
0.0 \%\end{array}$ \\
\hline & $\begin{array}{l}100 \% \\
0.0 \%\end{array}$ & $\begin{array}{c}96.7 \% \\
3.3 \%\end{array}$ & $\begin{array}{l}100 \% \\
0.0 \%\end{array}$ & $\begin{array}{c}98.3 \% \\
1.7 \%\end{array}$ & $\begin{array}{c}98.8 \% \\
1.2 \%\end{array}$ \\
\hline & 5- Note & $\begin{array}{c}10- \\
\text { Note }\end{array}$ & $\begin{array}{c}50- \\
\text { Note }\end{array}$ & $\begin{array}{l}100- \\
\text { Note }\end{array}$ & \\
\hline
\end{tabular}

B)

As shown in Tables 3 and 4, the performance of the ANN model using a denomination-specific ROIbased GLCM feature achieved a better result as compared to calculating the GLCM for the entire banknote. This result also supports the finding of Hasanuzzaman et. al [12].

Thirdly, the combinations of color and texture features in different arrangements are examined. From the experimental analysis, the study observed a performance improvement when the study considered the composite feature of color moments, dominant color, and Glcm feature with the order of Glcm followed by the dominant color and then color moments. Accordingly, the study observed a performance of 99.1, 99.1, and 99.6\% accuracy when considering a composite feature of Glcm followed by a dominant color and then color moment, color moments followed by a dominant color and then color moment, and dominant color which is followed by color moment and then Glcm feature to construct the composite feature respectively. From the experimental analysis, the arrangement we used to construct the composite feature would matter the recognition performance.

\section{a. Widen strip ROI extraction for banknote verification}

There are almost very tight similarities in between the surface of fake and genuine notes. It is almost impossible to study the banknote originality through color feature, rather it is required to identify the secured ROI that is not available in the fake notes. In doing so, for banknotes 50 and 100 there is a widening strip ROI security feature that is not mostly observed in the fake note. In a banknote recognition system, ground truth images for the front side of every category note are first collected under optimal conditions. The localized Golden strip ROI, which is computed from 50-note and 100-notes is depicted in Figure (6). 


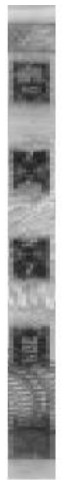

(A)

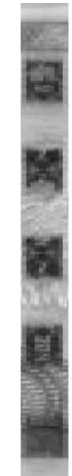

(B)

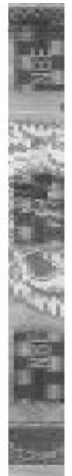

(C)

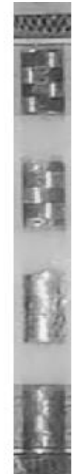

(D)

Figure 6. ROI calculated from different bill notes, A) from 10, B) from 50, C)100, and D) from 200-bill notes

Finally, the mean, standard deviation, and skewness of the three channels such as hue, saturation, and intensity of the HSV color space, the dominant color (the color with the highest pixel occupancy) and entropy calculated from the entire note, and the contrast, correlation, energy, and Homogeneity of Widen strip ROI and from six-block GLCM matrix were calculated and concatenating as a feature vector. The following confusion matrix table depicts the performance of the ANN model using the composite features (See Table 5).

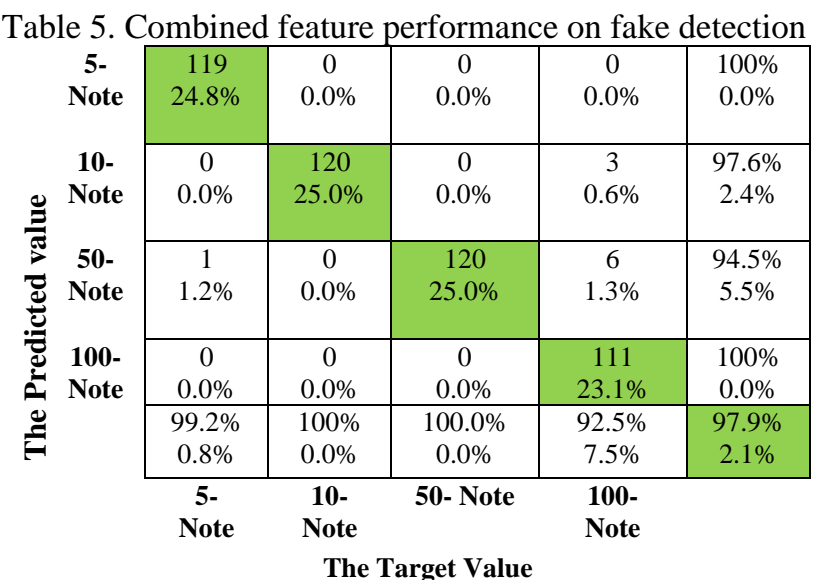

As shown in Figure 7, this depicts the performance of ANN model. Accordingly, the best training and validation performance is registered at epoch number 8 .

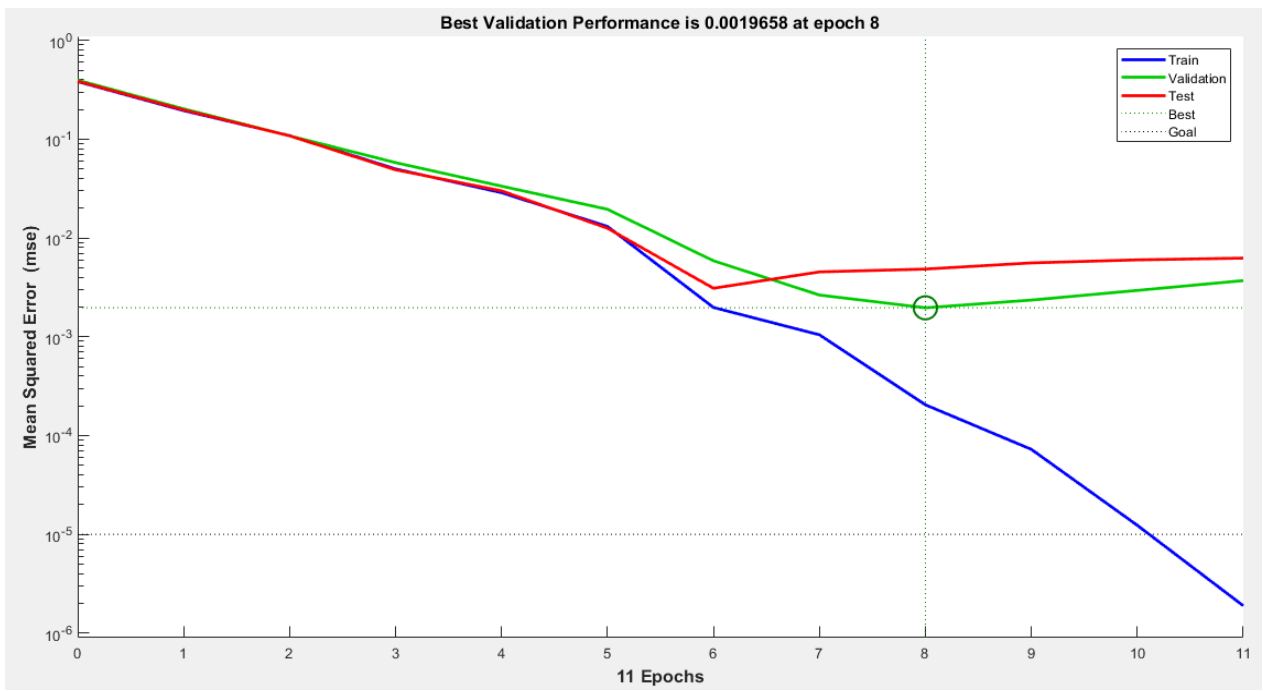

Figure 7. ANN training performance at epoch 16 


\section{CONCLUSION AND FUTURE WORK}

In this research, a banknote recognition method is proposed based on the denomination-specific ROI. In the study, the dominant color and denomination-specific ROI were identified and calculated. Following the color moments, contrast, correlation, energy, and homogeneity of the denomination-specific components, the entropy value of the ROI was calculated. Then, the different feature matrix was evaluated individually and together by different arrangements until better efficiency was achieved. A genetic algorithm was used used to maximize the computational efficiency thereby reducing the computational requirement of the model.

The proposed ANN-based recognizer has the advantages that: - it is robust and effective; it emphasizes the criticality it compensates for various geometric and lighting parameters. In addition, the denominationspecific ROI is applied to handle occluded, noisy, and old Ethiopian bill notes in handling old, folded, and occluded banknotes. It affords a valuable, reliable, and friendly tool for visually impaired people to easily recognize Ethiopian bill notes. Our experimental results using two-fold cross-validation on the Ethiopian currency dataset show that the proposed ANN-based banknote recognition method yields better accuracy.

Moreover, the proposed algorithm has been evaluated by datasets from a variety of conditions including occlusion, rotation, scaling, cluttered background, illumination change, viewpoint variation, and worn or wrinkled bills, and further by blind subjects. Our approach achieves a 98\% true recognition rate and 2\% negative recognition rate. Furthermore, studies are required to be conducted on the mobile-based banknote classification and recognition system that can enable physically impaired people to identify the note using their hand-held cell phone.

In the future work, we will address the issue of motion blur in two perspectives: 1) integrate deblurring techniques [6,7] into our system; 2) provide a simple training process to familiarize blind users with the device to reduce motion blur. Our future work will also focus on evaluating the proposed system to recognize banknotes of different countries and transferring the method to mobile phones.

\section{REFERENCES}

[1] AhmedKhan Tareen and Zahra Saleem, (International Conference on Computing, Mathematics and Engineering Technologies). BRISK features are invariant to scale, rotation, and limited affine changes. International Conference on Computing, Mathematics and Engineering Technologies.

[2] Alene, A. (2019). Deep Learning Approach for Ethiopian Banknote Denomination Classification and Fake Detection System. International Journal of Computer Science and Control Engineering, 30-37.

[3] Alphonsa Thomas, Sreekumar K. (2014). A Survey on Image Feature Descriptors Color, Shape and Texture. International Journal of Computer Science and Information Technologies.

[4] Anusha, Usha Reddy, and T. Ramashri. (2014). Content Based Image Retrieval using Color Moments and Texture. International Journal of Engineering Research and Technology, 3(2).

[5] Aoba, M.; Kikuchi, T.; Takefuji, Y. Euro banknote recognition system using a three-layered perception and RBF networks. IPSJ Trans. Med. Model. Its Appl. 2003, 44, 99-108. [Google Scholar]

[6] Beck A, Teboulle M. Fast Gradient-Based Algorithms for Constrained Total Variation Image Denoising and Deblurring Problems. IEEE Trans. Image Processing. 2009;18(11) [PubMed]

[7] Dong W, Zhang L, Shi G, Wu X. Image Deblurring and Super- Resolution by Adaptive Sparse Domain Selection and Adaptive Regularization. IEEE Trans. Image Processing. 2011;20(7) [PubMed]

[8] Faiz M. Hasanuzzaman, Xiaodong Yang, and YingLi Tian. (2012). Robust and Effective Component-based Banknote Recognition for the Blind. PMC, 24(2), 1021-1030

[9] Faiz M. Hasanuzzaman, Xiaodong Yang, and YingLi Tian. (2019, 8 23). Robust and Effective Component-based Banknote Recognition by SURF Features. New York, NY, 10031 , USA.

[10] García-Lamont, F.; Cervantes, J.; López, A. Recognition of Mexican banknotes via their color and texture features Expert System. Appl.2012,39, 9551-9660.

[11] Gaurav Kumar and Pradeep Kumar Bhatia. (2014). A Detailed Review of Feature Extraction in Image Processing Systems. Fourth International Conference on Advanced Computing \& Communication Technologies.

[12] Hasanuzzaman, F.M.; Yang, X.; Tian, Y.-L. Robust and effective component-based banknote recognition for the blind. IEEE Trans. Syst. Man Cybern. CAppl. Rev.2012,42.

[13] Hlaing, K. N. N., \& Gopalakrishnan, A. K. (2016). Myanmar paper currency recognition using GLCM and k-NN. In Second Asian Conference on Defence Technology (ACDT), (pp. 67-72). IEEE.

[14] Humeau Heurtier A. (2019). Texture Feature Extraction Methods: A Survey. IEEE Access. 7(2).

[15] Jegnaw Fentahun Zeggeye, Yaregal Assabie, "Automatic Recognition and Counterfeit Detection of Ethiopian Paper Currency," I.J. Image, Graphics and Signal Processing, 2016

[16] Kocur I, Parajasegaram R, Pokharel G. Global Data on Visual Impairment in the Year 2002. Bulletin of the World Health Organization. 2004; 82:844-851. [PMC free article] [PubMed]

[17] Lee, J.-K.; Jeon, S.-G.; Kim, I.-H. Distinctive point extraction and recognition algorithm for various kinds of euro banknotes. Int. J. Control Autom. Syst. 2004, 2, 201-206.

[18] Llive, C.; Roberto, Ch.; Ayala, G.; Andrés, C. Desarrollo e Implementación de un Software de Reconocimiento de Dólares Americanos Dirigido a Personas con Discapacidad Visual Utilizando Teléfonos Móviles Inteligentes con Sistema Operativo Android. Available online: http://repositorio.espe.edu.ec/xmlui/handle/21000/4752 (accessed on 5 April 2016). 
[19] M.-S. Choi and W.-Y. Kim. A novel two stage template matching method for rotation and illumination invariance. Pattern recognition, 35(1):119-129, 2002.

[20] R. C. Joshi, S. Yadav and M. K. Dutta, "YOLO-v3 Based Currency Detection and Recognition System for Visually Impaired Persons," 2020 International Conference on Contemporary Computing and Applications (IC3A), Lucknow, India, 2020, pp. 280-285, doi: 10.1109/IC3A48958.2020.233314.

[21] R. C. Joshi, S. Yadav, M. K. Dutta, and C. M. Travieso-Gonzalez, "Efficient Multi-Object Detection and Smart Navigation Using Artificial Intelligence for Visually Impaired People,” Entropy, vol. 22, no. 9, p.

[22] Rajkumar Goel, Vineet Kumar, Saurabh Srivastav, and K. Sinha. (2017). A Review of Feature Extraction Techniques for. International Journal of Advanced Research in Computer and Communication Engineering.

[23] Safraz, M. An intelligent paper currency recognition system. Proc. Comput. Sci. 2015, 65, 538-545

[24] Shefraw Asfaw Alene and Million Meshesha. (2019). Ethiopian Paper Currency Recognition System: An Optimal Feature Extraction. IEEE-SEM, 7(8).

[25] Sonali Bhagat and Sarika Patil. (2016). Indian currency recognition for visually disable people using image processing. International Journal of Advanced Research in Computer and Communication Engineering.

[26] Takeda, F.; Omatu, S. High speed paper currency recognition by neural networks. IEEE Trans. Neural Netw. 1995, 6 , $73-77$.

[27] Vila, A.; Ferrer, N.; Mantecon, J.; Bretón, D.; García, J.F. Development of a fast and non-destructive procedure for characterizing and distinguishing original and fake euro notes. Anal. Chim. Acta 2006, 559, 257-263.

[28] WBU (World Blind Union). Available online: http://www.who.int/mediacentre/factsheets/fs282/en/ (accessed on 28 April 2013).

[29] You Fu-cheng. Zhang Cong \&, The Technique of Color and Shape-based Multi- feature Combination of TradeMark Image Retrieval, 2010

[30] Young Ho, P.; Seung Yong, K.; Tuyen Danh, P.; Kang Ryoung, P.; Dae Sik, J.; Sungsoo, Y. A high-performance recognition system based on a one-dimensional visible light line sensor. Sens. J. 2015, 15, 14093-14115.

\section{BIOGRAPHY OF AUTHOR}

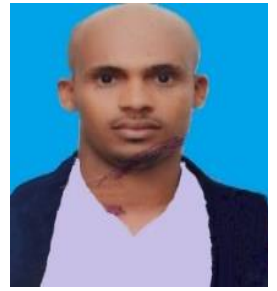

Asfaw Alene Shefraw received his BSc. degree and his Msc. degree in computer science, at Mekelle and Bahir Dar University respectively, Ethiopia, in Jun 2008 and July 2019 respectively. In addition, his Msc in visual communication design at UNIKOM, Bandung, Indonesia. He is currently works as a lecturer at computer science department, Bahir Dar University, Ethiopia. His current research focuses on recognition of objects, texture, and scene categories. His research interests include image processing, object detection, and developing effective image representations for recognition. 